\title{
Uplink packet scheduling in cellular networks with relaying-comparative study
}

\author{
D.C. Dimitrova $\cdot$ J.L. van den Berg $\cdot$ G. Heijenk
}

Published online: 27 May 2010

(C) The Author(s) 2010. This article is published with open access at Springerlink.com

\begin{abstract}
Deployment of intermediate relay nodes in cellular networks, e.g. UMTS/ HSPA, has been proposed for service enhancement, which is of particular importance for uplink users at the cell edge suffering from low power capacity and relatively poor channel conditions. In this paper, we propose and investigate a number of uplink packet scheduling schemes deploying the relay functionality in different ways. Using a combined packet and flow level analysis capturing the specifics of the scheduling schemes and the random behavior of the users (initiation and completion of flow transfers), the performance of the various schemes is evaluated and compared to a reference scenario where relaying is not used. The main performance measures considered in our study are realized data rates and mean flow transfer times. Interestingly, considering flow transfer times, it is found that the use of relay nodes is not only particularly beneficial for users at the cell edge but also has a strong, positive effect on the performance of users at other locations not transmitting via a relay node.
\end{abstract}

Keywords Relay $\cdot$ Scheduling $\cdot$ Flow level analysis

D.C. Dimitrova $(\varangle)$ J J.L. van den Berg · G. Heijenk

University of Twente, Postbus 217, 7500 AE Enschede,

The Netherlands

e-mail: d.c.dimitrova@ewi.utwente.nl

G. Heijenk

e-mail: geert.heijenk@ewi.utwente.nl

J.L. van den Berg

TNO ICT, Delft, The Netherlands

e-mail: j.l.vandenberg@ewi.utwente.nl

\section{Introduction}

In the last decade UMTS (Universal Mobile Telecommunications System) evolved from an on-paper standard to a full scale technology adopted by many operators around the globe. The system continues to advance towards improved radio resource efficiency and higher data rates with the HSPA (High Speed Packet Access) technologies being the latest upgrade. For the downlink, from base station to mobile, the HSDPA (High-Speed Downlink Packet Access) technology has been standardized by 3GPP in [1]. Alternatively, [2] introduces the EUL (Enhanced Uplink) for the uplink, from mobile to base station. In EUL, the channel resource is shared among all active users. Channel access is organized by the base station (BS) according to a particular scheduling scheme operating on a time scale of $2 \mathrm{~ms}$ (TTI-Transmission Time Interval).

In this paper we show the potential performance benefits of relaying for EUL, with focus on the resulting modifications on packet scheduling. In particular, we discuss six relay-enabled Round Robin schedulers and a reference scheme which does not use relaying. The schemes are compared with respect to a range of performance measures such as received powers, realized data rates and mean flow transfer times.

In recent years many studies concentrated on performance evaluation of various scheduling schemes for EUL, $[5,8]$ to mention some. In principle, independently of the scheme, remote mobile stations (MSs) suffer from low data rates due to high path loss. Some authors, see [11], propose service in parallel due to users' limited power capacity. However, this only improves resource utilization but not the offered service. Placing a relay station (RS) can positively influence data rates by breaking a long communication path MS-BS into two shorter paths, MS-RS and RS-BS namely. 
The idea of relaying is rather attractive, however its implementation is non trivial since it poses new implementation decisions such as where to set the relay or what scheduler to apply. As each decision has several possible outcomes the number of potential implementations rapidly grows. Selecting an optimal solution requires identifying main implementation choices as well as their advantages and disadvantages. Most importantly, before any research, we need to show that relaying can improve performance.

The application of relaying has been researched for a range of cellular and wireless networks. [13] discusses a downlink scenario with relays placed at $2 / 3$ of the cell radius. The authors shows that in the presence of interference, for a single MS, sending via the relay is beneficial in terms of packet errors and delays. The authors of [10] evaluate a LTE (Long Term Evolution) network with relays and changing number of MSs but evaluate only outage probability. Furthermore, the paper benefits from considering scheduling which adapts to channel conditions but lacks realism since unlimited flow sizes are assumed. Other studies which show the advantages of relaying for OFDMA networks are $[12,15]$, while [14] and [3] concentrate on WiMAX networks.

In [4] we presented a comparative analysis of several packet schedulers which incorporated relaying. In particular, all schemes use the radio interface for the communication between a mobile and relay station and between a relay and base station. The most prominent contributions of [4] were: incorporating the impact of flow level dynamics in terms of randomly changing number of active users and discussing variety of schedulers with relaying as opposed to the few options presented by other authors. The current study is an extension of [4] as we introduce several additional scheduling schemes, see Sect. 2. We are particularly interested in the deployment of relaying with semi-fixed infrastructure, in which the relay is connected to the base station via an existing infrastructure. The benefits and drawbacks of this approach are discussed in Sect. 2.

The paper continues as follows. First, in Sect. 2, we briefly discuss the relaying concept and describe the scheduling schemes considered in this paper. The model description and analysis appear in Sect. 3 and Sect. 4, respectively. Section 5 presents our findings of the performance evaluation. Finally, Sect. 6 summarizes our work.

\section{Relay-enabled scheduling schemes}

The key resource in EUL is the total power budget at the base station, which is shared among the mobile stations (MSs). A mobile station is an EUL compatible device which often operates on batteries and as such has limited transmit power. Depending on its distance to the BS a MS can or cannot use the total available resource on its own. The particular assignment of transmitted powers and TTIs over the active mobile stations depends on the scheduling scheme. Typically scheduling decisions are taken at the base station, which knows the number of ongoing flows. Introducing a relay station (RS) however requires modifications of the scheduler such that certain TTIs are dedicated to relay transmissions. We first briefly recall the idea of relaying in a cellular system and introduce the required notation. Next, the scheduling schemes considered in this paper are described.

\subsection{Relaying}

Relaying allows us to reduce signal degradation due to path loss by breaking up a long path into two short ones. However, these gains are against the cost of an additional transmission of the data at the relay node. In a relay-enabled system a MS chooses between direct communication to the BS (direct path MS-BS) and sending via the relay (relay path MS-RS-BS), depending on the data rates that can be realized on these paths. Note that the relay path consists of two sub-paths-MS-RS and RS-BS, and the data rate realized on the relay path depends on the achievable data rates on the two sub-paths.

Each (sub-)path is characterized by a set of transmission parameters: the distance between the communicating devices $d_{z z}$, the path loss $L_{z z}$, the transmit power $P_{z z}^{t x}$, the duration of a transmission opportunity $\tau_{z z}$ and the realized data rate $r_{z z}$ during a transmission opportunity. The index $z z$ refers to the specific (sub-)path, i.e. $m s$ for the direct path from MS to BS, $m r$ for the sub-path from MS to RS, and $r s$ for the sub-path from RS to BS. The transmission times $\tau_{m r}$ and $\tau_{r s}$ are scheduler specific; their sum is denoted by $\tau=\tau_{m r}+\tau_{r s}$. The relations between the transmission parameters are further discussed in Sect. 4 . We will now continue to introduce the specific scheduling schemes considered in our study.

\subsection{Scheduling schemes}

Scheduling decisions are taken by the BS for which a relay behaves as a stationary MS that needs to be scheduled. Several approaches towards incorporating relay transmissions into scheduling are possible. We consider only Round Robin (RR) type of schedulers where mobile users are served oneby-one (OBO), independently of their channel conditions. Studies among which $[9,11]$ show that OBO is inefficient in resource utilization when remote users with limited power capacity are served because remote users are limited in their realized data rates by their maximum transmitted power. However, a relay is closer to the MSs and it is more likely that a single MS is capable to fully use the available resources. 
In our study we consider five variants of relay-aware OBO schedulers for all of which the communication between MS and RS and between RS and BS uses the same radio interface, see Fig. 1(a). In addition, we also discuss a relay-enabled scheme where the relay station is connected to the base station via an (existing) fixed line, see Fig. 1(b). As a reference scheduler which allows us to evaluate the benefits of relaying a plain $O B O$ is considered. In OBO a MS always transmits directly to the base station independently of its location in the cell (and the duration of a single transmission opportunity equals one TTI, i.e. $\tau_{m s}=2 \mathrm{~ms}$ ). In the relay-enabled schemes a MS can select the direct or the relay path, depending on its location relative to the BS and the RS.

Below each of the considered scheme is presented providing more details on the specific power assignment and service distribution. Figure 2 shows graphical representation of the schemes. The $\mathrm{x}$-axis presents the time progress while the y-axix the received power at the next station. Note that the received power has a physical limitation $B$.

Extended $O B O(\mathrm{ExOBO})$ assigns (in the case an indirect path is chosen) a single TTI to the transmissions on both sub-paths, i.e. $\tau_{m r}=\tau_{r s}=2 \mathrm{~ms}$. Therefore, for the service of a particular mobile station in a single round of the RR scheme the base station sets aside 2 TTIs meaning $\tau=4 \mathrm{~ms}$. Transmissions on a direct path are assigned one TTI.

In the Shared $O B O$ (SOBO) scheme transmissions on both direct and indirect paths are assigned one TTI, i.e. $\tau=2 \mathrm{~ms}$. In the case of an indirect path, the single TTI is divided into two equal intervals of $1 \mathrm{~ms}$ and MS and RS

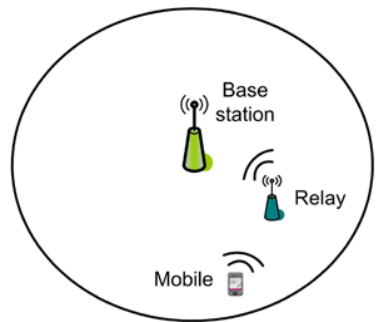

(a) Radio interface

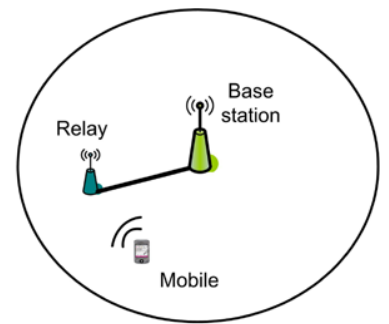

(b) Fixed infrastructure interface
Fig. 1 Deployment scenarios for relay-enabled scheduling: (a) with using only radio interface connections and (b) with a fixed-line connection between the base and relay station both receive one interval to transmit, i.e. $\tau_{m r}=\tau_{r s}=1 \mathrm{~ms}$. Assigning fixed-length transmission times of one TTI for direct and indirect paths eases implementation, but the static subdivision for indirect paths is inefficient when the achievable rates on the sub-paths differ.

Optimized SOBO (SoptOBO) also uses one TTI to serve a single MS, both for direct and indirect paths (i.e. $\tau=$ $2 \mathrm{~ms}$ ). However, for indirect paths, it selects the transmission times on the sub-paths MS-RS and RS-BS such that the same amount of data is transferred, i.e. $\tau_{m r} r_{m r}=\tau_{r s} r_{r s}$. Although SoptOBO maximizes the resource utilization it is rather challenging for implementation. The selection of the specific transmission times for the sub-paths requires more complex functionality in the base station most notably decoding and recoding the received signal.

In order to establish a best case performance for the SOBO and SoptOBO schemes we also designed two corresponding idealistic schemes, namely ideal SOBO (SOBOid) and ideal optimized SOBO (SoptOBOid). The SOBOid scheme adopts the same TTI division principle as SOBO, while SoptOBOid behaves as SoptOBO. For both schemes the assumptions apply that (i) the interference budget at the relay station and (ii) the transmitted power of the relay station are unlimited. The former assumption implies that mobile stations can realize their maximum achievable data rate to the relay station, given their maximum transmitted power. The latter assumptions has the consequence that the relay can, independently of its location, fully utilize the budget at the base station. Under these assumptions, the interference budget and maximum transmitted power of the relay are eliminated as a potential bottleneck. As result of the assumptions the schemes are more of a theoretical design rather than a practical solution but they can provide us some notion of best-case performance.

The last relay-enabled scheme in our analysis, namely FixedRS, makes use of a fixed-line connection between the relay and base station. Using a fixed-line connection has the advantage that transmissions from relay station to base station do not take resources away that can be used for transmissions by the mobile station to either relay station or base station. Of course, there will be an additional cost in connecting the relay station with the base station. This could for instance be realized using a wireline connection (e.g. fiber),

Fig. 2 Scheduling schemes

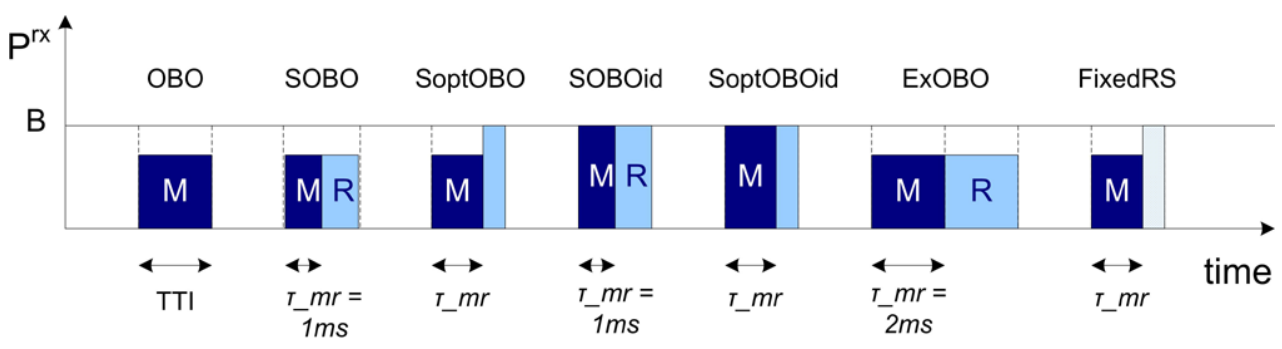


or using a different (fixed) wireless technology such as a microwave connection. Such line generally will generally have a limited capacity $C$, which is typically sufficiently high to allow for optimal utilization of the more costly radio interface. In line with the other schedulers, we will assume here that the data sent by a user will have arrived at the base station at the end of a TTI, to allow the BS to have complete information and control regarding the scheduling. Therefore the last fraction of the TTI $\left(\tau_{r s}\right)$ will be used by the RS to transmit the data it has received from the MS during the first $\tau_{m r}$ seconds to the base station, such that $\tau_{m r} r_{m r}=\tau_{r s} C$.

\section{Model}

Our model consists of a single cell with EUL users (MSs) generating data traffic. In order to differentiate in the location of MSs we divide the cell in $K$ zones with equal area, see Fig. 3. Each zone is characterized by its distance to the base station $d_{i}$, measured from the outer edge of the zone, and a corresponding path loss $L_{i}, i=1, \ldots, K$. All MSs within the same zone $i$ are assumed to have the same distance $d_{i}, i=1, \ldots, K$ to the base station. The distribution of (active) MSs over the zones is represented by the system state vector $\underline{n} \equiv\left(n_{1}, n_{2}, \ldots, n_{i}, \ldots, n_{K}\right)$, where $n_{i}$ is the number of currently active users (MSs) located in zone $i$.

Mobile stations become active, at uniformly distributed positions in the cell, according to a Poisson process with rate $\lambda$, and have to transfer a file with mean size $F$ (the file size has an exponential distribution). Hence, due to the equal area zones, all zones have the same file transfer initiation rate (or: arrival rate) $\lambda_{i}=\lambda / K$. Active MSs keep their position during the file transfer. A MS chooses between direct transmission or transmissions via the relay, depending on its location. All mobile stations have the same maximum transmit power capacity $P_{\max }^{t x}$. Depending on their location either the maximum transmit power or a lower power is used, i.e.

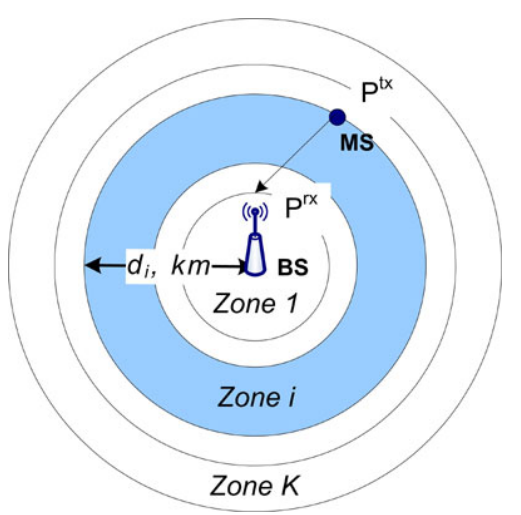

Fig. 3 Single cell model with cell division in zones. The distance between MS-BS, MS-RS and RS-BS are denoted as $d_{m s}, d_{m r}$ and $d_{r s}$, respectively
$P^{t x} \leq P_{\max }^{t x}$, such that a MS maximizes its utilization of the available budget $B$.

In our study a relay is an amplify-and-forward device with one basic functionality, namely resending data from MS to BS. Such simple design keeps the cost down and still provides improved performance to the MSs.

In this paper we assume that a mobile station always lays on a straight line with a relay and the base station. ${ }^{1} \mathrm{~A}$ fixed path selection policy based on distance is adopted according to which all MSs located beyond the relay, i.e. $d_{m s}>d_{r s}$, transmit via the indirect path and in all other cases, i.e. $d_{m s} \leq d_{r s}$, direct transmission is chosen. An ideal switching mechanism between transmitting and receiving at the RS is assumed as the same frequency band is used. In the special case of FixedRS scheduler the line connecting the relay and base station has by default very high capacity such that it never forms the bottleneck on the indirect path. We consider all technological issues related to the fixed line to be transparent for our analysis.

At both BS and RS, limited channel resource, budget $B$, is assumed. Given noise rise $\eta$ and constant thermal noise $N$, the shared budget $B$ at the BS is derived: $B=\eta \times N$. The same budget $B$ is set also for the RS, with the exception of SOBOid and SoptOBOid for which the RS budget is unlimited. The other interference component we account for is the self-interference, i.e. the own signal parts which travel via secondary (reflected) paths. Intentionally disregarding important factors such as inter- and intra-cell interference allows us to identify the effect relaying has on performance. However, we realize that such factors may have negative effects on the performance gains.

\section{Analysis}

Relaying has two opposite effects on performance (excluding effects outside the scope of this paper): on the one hand it enables a higher received power level (increasing the data rate), but on the other hand forwarding at the RS requires an additional transmission of the data (decreasing effective data rates). Apart from that performance is influenced by the varying number of active users in the cell. In order to account for these effects we apply a combined packet and flow level analysis. For each scheduler we start with calculating received powers from which subsequently data rates can be derived. Next we consider flow level dynamics, e.g. flow initiation and flow completion, and model the system by a

\footnotetext{
${ }^{1}$ In fact, this assumption requires unlimited number of relays on a concentric circle but it can be shown that this ideal situation can be very well approximated by 8 or $10 \mathrm{RS}$. In principle, the analysis in this paper can easily be extended to any other assumption concerning the number and position of the RSs. However, for the purpose of the present paper, this specific choice suffices.
} 
continuous time Markov chains (CTMC) with transmission rates derived from the instantaneous data rates and the system state $\underline{n}$. From the steady state distribution of the Markov chain the mean flow transfer time of a MS in a particular zone can be obtained. Our combined approach exhibits several advantages above other existing approaches. Starting at the packet level allows us to catch specifics of scheduling scheme and environment. Applying Markov models supports the modelling of a real network where the number of active users constantly changes. The approach is also rather scalable since changing the scheduler or the environment only ask for recalculation of the instantaneous rate at the packet level.

\subsection{Received powers}

According to the propagation law, the received power $P^{r x}$ is a direct result of applied transmit power $P^{t x} \leq P_{\max }^{t x}$ and path loss $L(d)$. Furthermore, the maximum possible received power from any MS is limited by the available budget $B$ resulting in:

$P^{r x}=\min \left(P^{t x} / L(d) ; B\right)$.

The assumed path loss model, taken from the Okumura-Hata model [7], is given by $L(d)=123.2+35.2 \log 10(d)$ (in $\mathrm{dB}$ ) with $d$ the distance in kilometer.

In Sect. 2 we explained how each scheduling scheme organizes its transmissions. Furthermore, when relaying is used, we should differentiate between the received powers $P^{r x}(B S)$ and $P^{r x}(R S)$ at the base station and the relay station, respectively.

When a relay is used in SOBO, SOBOid and ExOBO the slower sub-path limits the data rate. Therefore, the transmit powers at MS and RS are chosen such that the received powers at RS and $\mathrm{BS}$ are equal $\left(P^{r x}(R S)=P^{r x}(B S)\right)$ and determined by the minimum of the achievable received powers on both sub-paths. In the case of SoptOBO $P^{r x}(R S)$ is in general not equal to $P^{r x}(B S)$ since the scheme deploys different data rates on the two relay sub-paths. Note that SOBOid and SoptOBOid differ from the rest in the unlimited maximum transmission powers of RS it uses. The scheme-specific received powers can be given as:

$$
P^{r x}=\left\{\begin{array}{l}
P_{m s}^{r x} \quad M S-B S: \text { all schemes, } \\
\min \left(P_{m r}^{r x}, P_{r s}^{r x}\right) \\
\quad M S-R S-B S: \text { SOBO, SOBOid, ExOBO, } \\
P_{r s}^{r x} \quad \text { RS-BS: SoptOBO, SoptOBOid, } \\
P_{m r}^{r x} \quad \text { MS-RS: SoptOBO, SoptOBOid, FixedRS. }
\end{array}\right.
$$

\subsection{Instantaneous rate}

The data rate achieved on a (sub-) path $z z$ considering only the transmission channel conditions, i.e. received power and interference, is the instantaneous rate $r_{z z}$. Hence, this is the rate realized during $\tau_{m r}$. The instantaneous rate of a particular MS is given by:

$r_{z z}=\frac{R_{\text {chip }}}{E_{b} / N_{0}} \cdot \frac{P_{z z}^{r x}}{N+(1-\omega) P_{z z}^{r x}}$.

In the above equation $R_{\text {chip }}$ is the system chip rate and $E_{b} / N_{0}$ is the energy-per-bit to noise ratio. The index $z z=$ ( $m s, m r, r s$ ) refers to the (sub-)path. Note that for FixedRS $r_{r s}=C$ holds. The maximum possible data rate a MS can realize is determined by the condition that the budget $B$ can be filled, i.e. $P_{z z}^{r x}=B$.

\subsection{Effective rate}

The effective rates $r_{\text {eff }}$ accounts for the effects of relaying and is the rate realized by a MS for the duration of the TTI. On the direct path the effective rate is the same as the instantaneous, i.e. $r_{e f f}=r_{m s}$, because the entire TTI is used by the MS. On the indirect path however, due to data forwarding, the effective rate is lower than the instantaneous and depends on what part of the TTI is used by the mobile, i.e. on $\tau_{m r}$. Given the scheduler specific time assignment policy, $r_{e f f}$ can be derived from the instantaneous rate as:

$r_{e f f}=\left\{\begin{array}{l}r_{m s} \quad M S-B S: \text { all schemes, } \\ \min \left(r_{m r}, r_{r s}\right) * \frac{1}{2} \\ \quad M S-R S-B S: \text { SOBO, SOBOid, ExOBO, } \\ \frac{r_{m r} * \tau_{m r}}{\tau} \\ \quad M S-R S-B S: \text { SoptOBO, SoptOBOid, FixedRS. }\end{array}\right.$

SOBO, SOBOid and ExOBO assign to the mobile station only half of the total time $\tau$, since the factor $1 / 2$ in (4). Note that in ExOBO $\tau$ is equal to two TTIs. In SoptOBO, SoptOBOid and FixedRS $0<\tau_{m r}<$ TTI holds depending on the MS's location such that $r_{m r} \tau_{m r}=r_{r s} \tau_{r s}$ and $\tau_{m r}+\tau_{r s}=$ TTI.

\subsection{Flow dynamics}

The actual rate a MS receives is lower or equal to the effective rate $r_{e f f}$ and depends on the state $\underline{n}$ of the system and on the number of users $n$ in particular. This new rate we term state-dependent throughput $R(\underline{n})$ and it differs per 
Table 1 Relation between zone numbers and distances

\begin{tabular}{lllllllllll}
\hline Zone number & 1 & 2 & 3 & 4 & 5 & 6 & 7 & 8 & 9 & 10 \\
\hline Distance, $\mathrm{km}$ & 0.632 & 0.894 & 1.095 & 1.264 & 1.414 & 1.5492 & 1.673 & 1.788 & 1.897 & 2.000 \\
\hline
\end{tabular}

scheduler. In all schemes but ExOBO the state-dependent throughput can be derived from:

$R(\underline{n})=r_{e f f} / n$.

The difference for ExOBO originates from the different number of TTIs assigned to MSs using the direct and the relay path-one and two TTIs respectively. Hence, for $R(\underline{n})$ we can write:

$R(\underline{n})= \begin{cases}\frac{r_{\text {eff }}}{n+n_{\text {relay }}} & \text { for } M S s \text { using direct path, } \\ \frac{2 r_{\text {eff }}}{n+n_{\text {relay }}} & \text { for MSs using relay path, }\end{cases}$

where $n_{\text {relay }}$ is the number of MSs which make use of the relay station. Note that $r_{e f f}$ is different for each path according to (4).

The changes in the system state $\underline{n}$ (due to flow initiations and completions) are described by a continuous time Markov chain of $K$ dimensions with transition rates $\lambda_{i}$ and $R(\underline{n})$. The particular form of the Markov chain is scheduler specific via $R(\underline{n})$. A more detailed description of such CTMC can be found in [5]. All schemes but ExOBO are a multi-class $\mathrm{M} / \mathrm{G} / 1$ processor sharing (PS) models whose steady state distribution is readily available, e.g. [6]. For the ExOBO model we implemented a Markov chain simulator which, based on large number of state transitions, i.e. 1 million, provides us with the steady state distribution. Subsequently, we can derive performance measures such as mean flow transfer times $T_{i}$ :

$T_{i}=\frac{F}{r_{e f f, i}} * \frac{1}{1-\rho}$,

where $\rho=\sum \rho_{i}$ is the system load and $\rho_{i}=\lambda_{i} / r_{\text {eff,i }}$ is the load in a particular zone $i$. Note that all presented parameters are zone specific and thus depend on the zone, in which the MSs is located.

\section{Numerical results on relaying}

In this section we present a quantitative evaluation of performance at both packet and flow level for the discussed scheduling schemes. We are particularly interested in comparing performance based on measures such as received powers, energy consumption, instantaneous rates and mean flow transfer times. Special attention is given to the impact of flow level dynamics. Most results are generated from mathematical analysis with small part coming from simulations, i.e. flow level analysis of ExOBO. Simulations are carried out with a generic simulator developed in Matlab for deriving the steady-state distribution of multi-dimensional Markov chains.

\subsection{Parameter settings}

In the numerical experiments we apply a system chip rate $R_{\text {chip }}$ of $3840 \mathrm{kchips} / \mathrm{s}$, thermal noise level $N$ of $-105.66 \mathrm{~dB} \mathrm{~m}$ and noise rise target $\eta$ of $6 \mathrm{~dB}$, see [7]. For SOBOid and SoptOBOid the available budget $B$ at the RS is unlimited as well as the transmitted power of the relay. The capacity of the fixed link in FixedRS is by default $10 \mathrm{Mbps}$. Self-interference of $10 \%$ is considered, i.e. $\omega=0.1$.

The cell is divided in $K=10$ zones with equal area $^{2}$, see Fig. 1. Given a cell radius of $2 \mathrm{~km}$ we applied straightforward link budget calculations to determine the zone radii, taken from the outer circle, and the path losses, see Table 1. In the rest of the paper for simplicity we talk about zone numbers as in the presented figures the zones are reflected by markers.

By default relay stations are located at $1.09 \mathrm{~km}$ (zone 3) from the BS with some experiments explicitly stating when this changes. Both MS and RS have maximum transmit power $P_{\max }^{t x}=0.125$ Watt. EUL flows are taken with an $E_{b} / N_{0}$ target of $5 \mathrm{~dB}$ [7] and mean file size is $F=1000$ kbit. The flow arrival rate is set to $\lambda=0.4$ flows $/ \mathrm{sec}$.

\subsection{Received power levels}

In order to build up good understanding of the impact relaying has on schedulers, we confine the discussion of received powers to OBO, SOBO and ExOBO. The received powers at the $\mathrm{BS}$ as a function of the distance are presented in Fig. 4 for a range of positions of the RSs. As expected, relaying increases received powers but the position of the RS has strong impact on the gain. Moving the RS towards the edge of the cell lowers the gain - an effect caused by increased distance $d_{r s}$ between RS and BS and fewer MSs using the relay.

Recall that transmission on the relay path can be limited by both relay and mobile station, which is illustrated by the graphs for RSs in zone 1 and 2 in Fig. 4. Typically the received power degrades with distance as we can see from the graph of direct transmission, i.e. OBO. However, when the RS forms the bottleneck on the relay path, all MSs served by it are characterized by the same received powers at the BS

\footnotetext{
${ }^{2}$ Extensive numerical experiments showed that this granularity is sufficient for our purposes.
} 
Fig. 4 Received powers at the base station for $\mathrm{OBO}$ (direct transmissions) and SOBO (relay-supported transmissions)

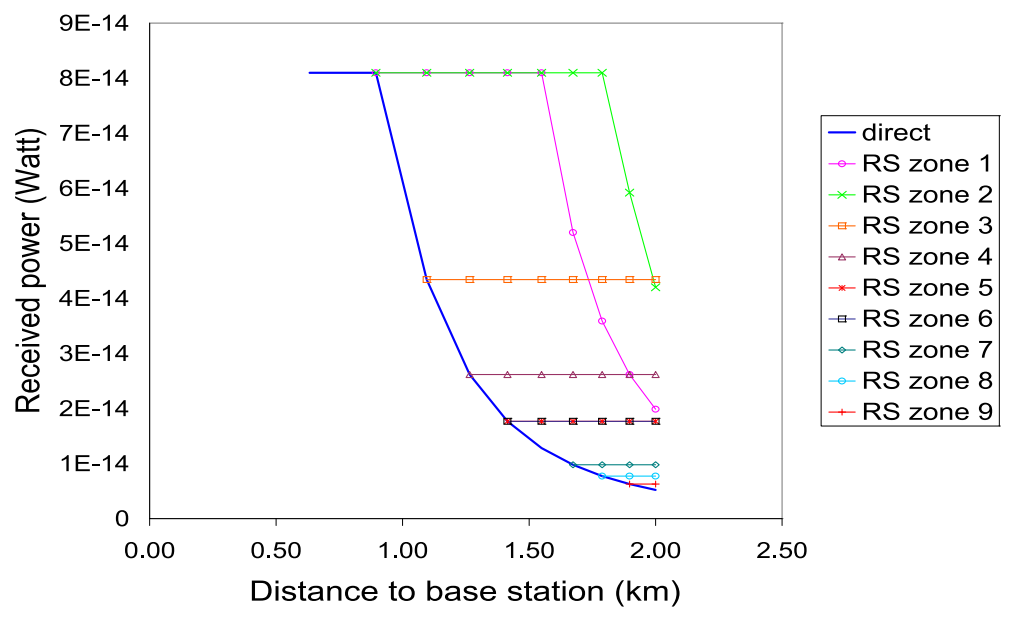

which explains the unchanging graphs. Note that with RS in zone 3 and further this is always the case. If the MSs are the bottleneck, the received powers decrease with distance and differ per zone.

\subsection{Effective data rates}

The effective data rates generated by each of the 'realistic' scheduling schemes are presented in Fig. $5 .^{3}$ The relay station is fixed at zone 3 and fixed path selection policy is applied, i.e. all mobile stations from zone 4 to 10 make us of relaying. In all cases when direct transmission is used all schemes show the same performance. However, beyond zone 3 (after the relay is used) the relay-enabled schemes outperform OBO, meeting our expectations. Only for SOBO and ExOBO the rates realized by users in zone 4 are actually lower when compared to OBO. This result illustrates very well the negative impact of relaying on transmission time due to data forwarding. Hence, we can conclude that relaying is generally effective but should be selected only when the indirect path delivers higher effective, and not instantaneous, rate than the direct one.

The graphs of SOBO and ExOBO coincide, since both schemes have the same received powers at BS and use only half the transmission time $\tau$ to transfer unique data, see (4). Furthermore, complying with our expectations, the two schemes are outperformed by SoptOBO. The flexible adaptation of the transmission times $\tau_{m r}$ and $\tau_{r s}$ supports the best possible utilization of the available budget. The FixedRS scheme offers further improvement since it in fact applies the same TTI division strategy, but the capacity of the RS-BS link is much higher than on the radio interface. Actually, the higher effective rates for FixedRS after the relay switches in imply that even mobile stations in front of the

\footnotetext{
${ }^{3}$ In the paper [4] the graphs of SoptOBO and SOBOid are erroneously exchanged. The current paper presents the proper graphs.
}

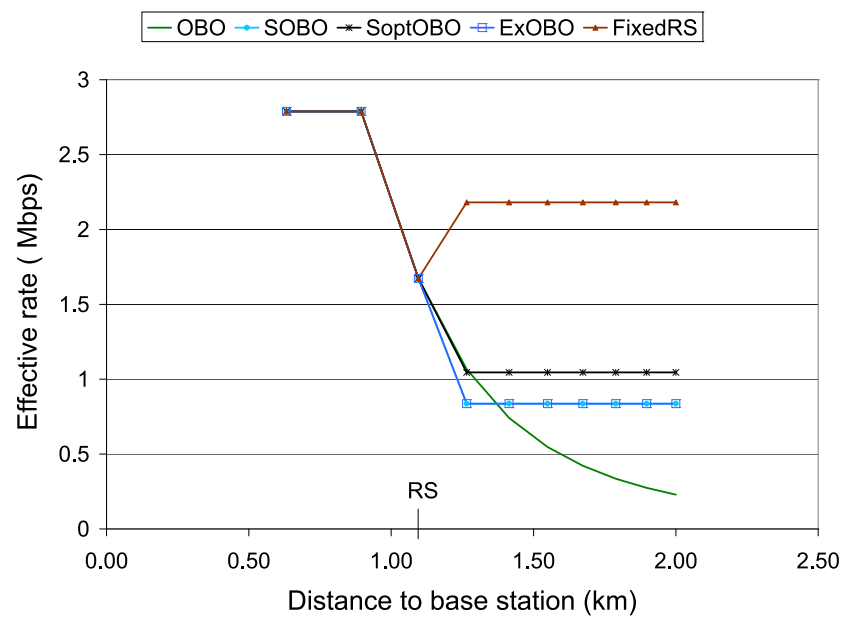

Fig. 5 Effective data rates realized by a non-relay OBO scheduler and by relay-enabled schemes: SOBO, SoptOBO, ExOBO and FixedRS

relay might benefit from its use. We explain that with the fact that users from zone 3 lack capacity to fully utilize the budget at the BS but can fill up the budget at the RS. Since the fixed link does not form a bottleneck, users from zone 3, if sending via the relay are able to fill up the budget at the BS as well.

Furthermore, in OBO the rate degrades with distance due to the differentiated service offered to users from different zones. Alternatively, given a relay in zone 3, all transmissions on the relay path in SOBO, SoptOBO, ExOBO and FixedRS are limited by the relay which explains the flat graphs.

A comparison between 'realistic' and 'idealistic' schemes is given in Fig. 6. As we can expect no limitations on the relay station result in better performance. It is more interesting to observe the effect of the TTI division strategy on performance. In SOBOid where the TTI is divided into two equal parts the relay forms the bottleneck on the indirect path thus causing the same performance of all MSs which use the relay. However, in SoptOBOid, in which the TTI division is 


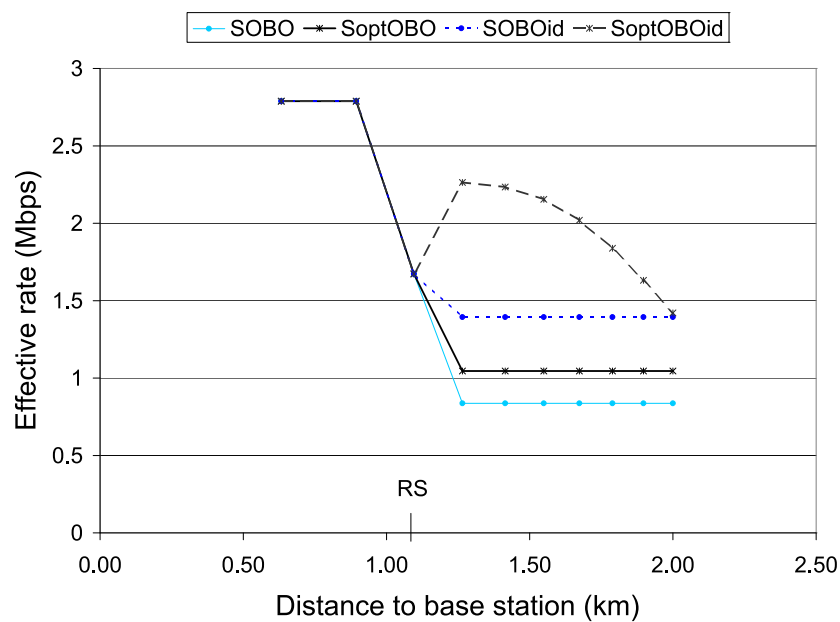

Fig. 6 Comparison between a realistic and idealistic scheduling realization, i.e. SOBO vs SOBOid and SoptOBO vs SoptOBOid

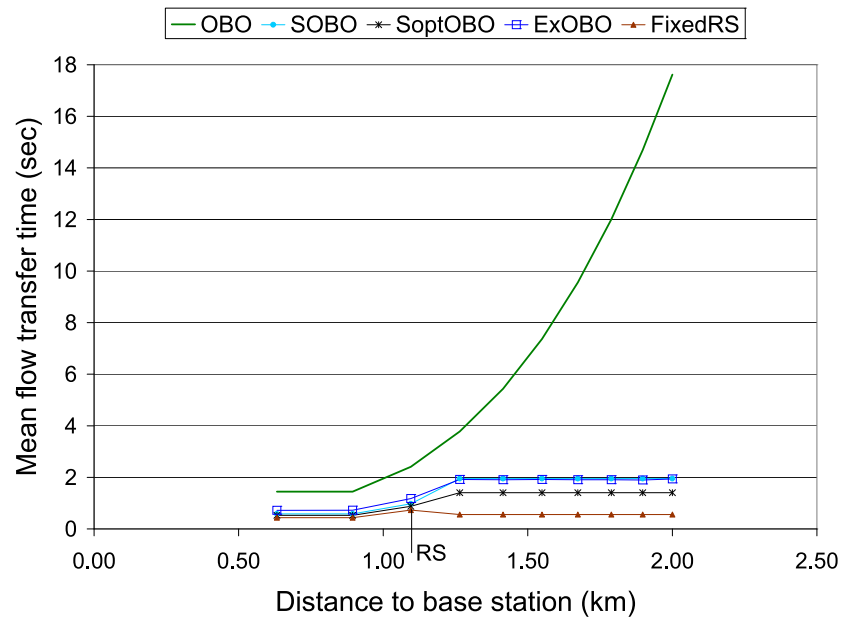

Fig. 7 Mean flow transfer times realized by a non-relay OBO scheduler and by relay-enabled schemes: SOBO, SoptOBO, ExOBO and FixedRS

adapted to match the capacity of the mobile stations, effective rates are changing according to signal propagation, i.e. the longer the distance the weaker the signal.

\subsection{Mean flow transfer times}

Figure 7 presents the dependency of the mean flow transfer times on the distance to the base station for the following relay-enabled schemes-Sobo, SoptOBO, ExOBO and FixedRS - and for the reference OBO scheduler. The observations made for the effective rates in Sect. 5.3 still hold, i.e. (i) $\mathrm{OBO}$ is the worst performing scheme; (ii) flexible TTI division, e.g. SoptOBO, provides benefits over fixed division, e.g. SOBO and ExOBO; and (iii) the higher capacity of the fixed line in FixedRS puts him as the best performing scheme.

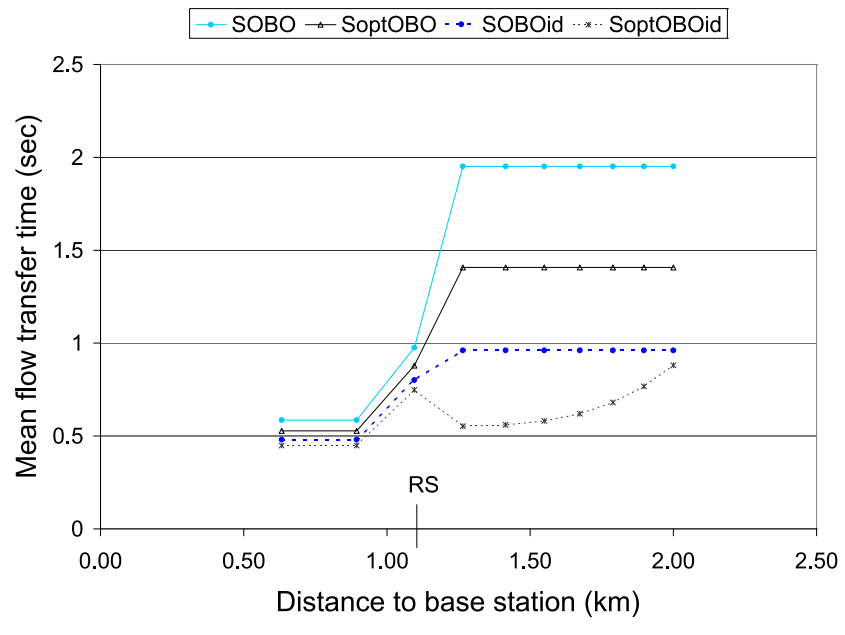

Fig. 8 Comparison between a realistic and idealistic scheduling realization, i.e. SOBO vs SOBOid and SoptOBO vs SoptOBOid

New trends in the performance exhibit as well. In particular, Fig. 7 shows that the gain achieved by using RSs is even more emphasized when we consider the mean flow transfer times as a performance measure. An interesting new observation is that even the performance of close-by MSs, which use direct transmissions, is improved as result of relaying. Higher data rates for remote MSs translate to lower system load (number of active MSs $n$ ), which is beneficial for all active MSs, see (6), independently whether they use a relay or not.

Again a comparison with idealistic transmission conditions at the RS is presented, see Fig. 8. The same trends as in the one for the effective rates can be made. The combined effect of flexible TTI division and distance on performance continues to be observable in the case of SoptOBOid. Also here the difference in performance is not only for MSs which use the relay but also for such stations which transmit via the direct path.

From the observations made in this and the previous section we could conclude that, next to FixedRS, SOBOid and SoptOBOid are the best performing schemes. Such conclusion will be misleading however because these schemes are rather idealistic and do not represent a real-world relay station. We have used them only to show the theoretical upper bound on the performance benefits provided by relaying.

\subsection{Capacity of fixed line}

We have varied the capacity of the fixed line connection between the BS and RS in the case of the FixedRS scheme. The results are presented in Fig. 9. The first observation, although somehow expected, is that increasing the link capacity leads to improved performance. The lowest link capacity of $2 \mathrm{Mpbs}$ lays very close to the capacity of a radio interface link. In this case the performance of the FixedRS scheme is 


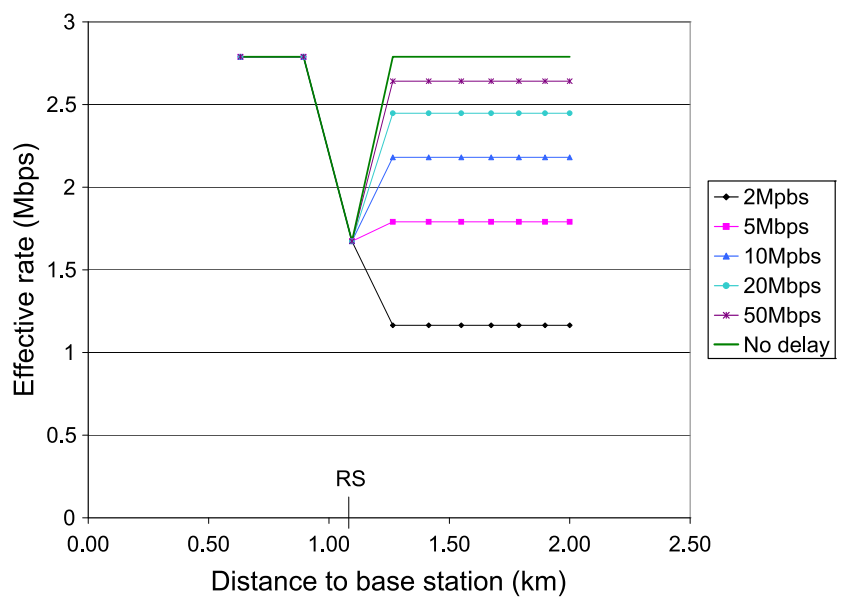

Fig. 9 The impact of the capacity of fixed line on the performance gain provided by relaying

comparable with the performance of the SoptOBO scheme. An upper bound is determined by the situation when the fixed link has zero delay and in fact the MSs can realize the maximum achievable effective rate given their limited transmitted power capacity. This is observable in Fig. 9 in the same effective rate values realized by direct and indirect transmission with the exception of zone 3 users which cannot fill up the budget.

Another interesting observation is that by increasing the fixed-line capacity not only the performance of mobile stations improves but also it quickly outperforms direct transmissions. Already with a $5 \mathrm{Mbps}$ line all MSs served via the relay receive better service than MSs in zone 3, which use the direct line but are limited by their own location. We can conclude that it might be advantageous to serve via the relay also MSs that are closer to the base station.

\section{Conclusion}

We have compared the performance of six Round Robin packet schedulers for uplink transmission in a cellular network with relaying. As performance measures we used received powers at the base station, instantaneous data rates and mean flow transfer times. Analysis of the flow transfer times (taking into account that the number of active users varies over time) is enabled by a combined packet- and flow level analysis.

Our study reveals the pros and cons of the six relay-aware scheduling schemes, and makes a comparison to the performance achieved in the case without relaying. As expected, relaying significantly improves the service offered to remote users with poor channel conditions. Interestingly, it is also beneficial for users who do not use the relay-an effect that can be seen only at the flow level. The latter observation supports our claim that the flow level dynamics have crucial influence on scheduling performance, and needs to be taken into account in performance comparisons.

As topics for further study we suggest investigating alternative relay-aware schedulers (e.g. with parallel transmissions), and examining the mutual relay influence in a more complex scenario with multiple cells.

Acknowledgements The authors would like to thank Remco Litjens for the prolific discussions and for providing us with challenging new ideas.

Open Access This article is distributed under the terms of the Creative Commons Attribution Noncommercial License which permits any noncommercial use, distribution, and reproduction in any medium, provided the original author(s) and source are credited.

\section{References}

1. 3GPP TS 25.308. High speed downlink packet access (HSDPA); Overall description.

2. 3GPP TS 25.309. FDD enhanced uplink; Overall description.

3. Bel, A., \& Lopez Vicario, G. S.-G. J. (2008). The benefits of relay selection in wimax networks. In ICT-MobileSummit '08.

4. Dimitrova, D., van den Berg, H., \& Heijenk, G. (2009). Performance analysis of uplink packet schedulers in cellular networks with relaying. In IFIP wireless and mobile networking WMNC'09 (pp. 263-273), Sept. 2009.

5. Dimitrova, D. C., van den Berg, H., Heijenk, G., \& Litjens, R. (2008). Flow-level performance comparison of packet scheduling schemes for UMTS EUL. In WWIC '08, vol. 5031. Tampere, Finland.

6. Haverkort, B. (1999). Performance of computer communication systems. New York: Wiley.

7. Holma, H., \& Toskala, A. (2006). HSDPA/HSUPA for UMTS. New York: Wiley.

8. Liu, T., Mäder, A., Staehle, D., \& Everitt, D. (2007). Analytic modeling of the UMTS enhanced uplink in multi-cell environments with volume-based best-effort traffic. In IEEE ISCIT '07, Sydney, Australia, Oct. 2007.

9. Ramakrishna, S., \& Holtzman, J. M. (1997). A scheme for throughput maximization in a dual-class CDMA system. In ICUPC '97, San Diego, USA.

10. Reetz, E., Hockmann, R., \& Tonjes, R. (2008). Performance study on cooperative relaying topologies in beyond $3 \mathrm{G}$ systems. In ICTMobileSummit '08.

11. Rosa, C., Outes, J., Sorensen, T. B., Wigard, J., \& Mogensen, P. E. (2004). Combined time and code division scheduling for enhanced uplink packet access in WCDMA. In IEEE VTC '04 (Fall), Los Angeles, USA.

12. Schoenen, R., Halfmann, R., \& Walke, B. H. (2008). An FDD multihop cellular network for 3GPP-LTE, pp. 1990-1994, May 2008.

13. Umlauft, M. (2006). Relay devices in umts networks-effects on. In Proceedings of the fifth annual Mediterranean ad hoc networking workshop (Med-Hoc-Net 2006).

14. Vidal, J., Munoz, O., Agustin, A., Calvo, E., \& Alcon, A. (2008). Enhancing 802.16 networks through infrastructure-based relays. In ICT-MobileSummit ' 08 .

15. Wei, H. Y., Ganguly, S., \& Izmailov, R. (2004). Ad hoc relay network planning for improving cellular data coverage. In Personal, indoor and mobile radio communications, 2004. PIMRC 2004 (vol. 2, pp. 769-773), Sept. 2004. 


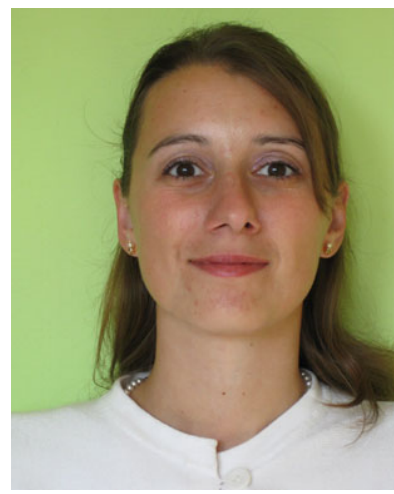

D.C. Dimitrova is currently completing her Ph.D. studies at the University of Twente, The Netherlands. She completed her B.Sc. studies Telecommunications at the Technical University of Sofia, Bulgaria and subsequently her M.Sc. degree in Telematics at the University of Twente, The Netherlands. Her major topic of research during the $\mathrm{Ph} . \mathrm{D}$. programme is scheduling in mobile cellular networks as she is interested in the trend of cooperation between wireless networks and the future development of such hybrid networks.

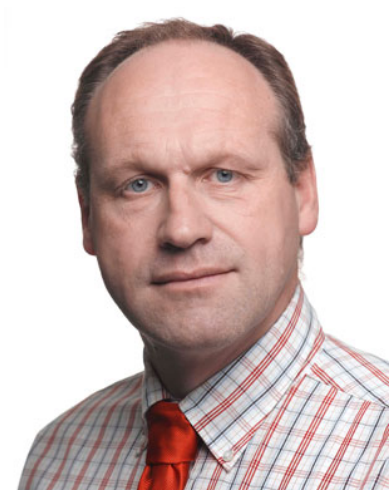

J.L. van den Berg is Senior Research Fellow and leader of the QoS group within TNO ICT. He received the M.Sc. and Ph.D. degree in mathematics from the University of Utrecht. From 1986, he worked at the Centre for Mathematics and Computer Science (CWI), Amsterdam. In 1990, he joined KPN Research (TNO ICT, since January 2003). The focus of his research is on planning, performance and QoS provisioning issues for mobile networks and service platforms. He has been active in numerous national and European research programs. Since July 2003 he has a part-time position as full professor in the department Design and Analysis of Communication Systems at the University of Twente.

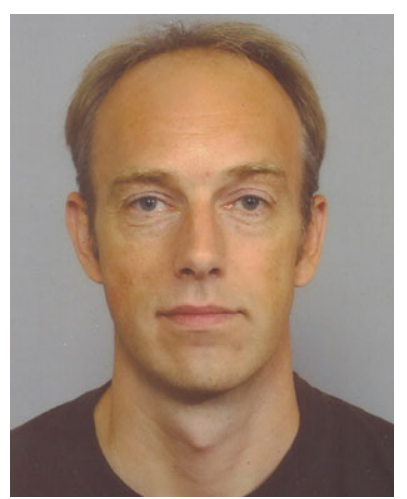

G. Heijenk received his M.Sc. in Computer Science from University of Twente, the Netherlands, in 1988. He has worked as a research staff member at the same university and received his Ph.D. in Telecommunications in 1995 . He has also held a part-time position as researcher at KPN research, the Netherlands, from 1989 until 1991. From 1995 until 2003, he was with Ericsson EuroLab Netherlands, first as a senior strategic engineer, and from 1999 as a research department manager. From 1998 until 2003 he was also a part-time senior researcher at the University of Twente. Currently, he is a full-time associate professor at the same university. Geert Heijenk has been a visiting researcher at University of Pennsylvania, Philadelphia and a visiting associate professor at University of California, Irvine. He is a senior member of the IEEE. His research interests include mobile-, wireless-, and ad-hoc networks, with a focus on sensor and vehicular networks. 